\title{
Existence results of fractional integro-differential equations with $m$-point multi-term fractional order integral boundary conditions
}

\author{
Weerawat Sudsutad ${ }^{1}$ and Jessada Tariboon ${ }^{1,2^{*}}$
}

"Correspondence:

jessadat@kmutnb.ac.th

'Department of Mathematics,

Faculty of Applied Science, King

Mongkut's University of Technology

North Bangkok, Bangkok, 10800,

Thailand

${ }^{2}$ Centre of Excellence in

Mathematics, CHE, Sri Ayutthaya

Road, Bangkok, 10400, Thailand

\begin{abstract}
In this article, we present some new existence and uniqueness results for nonlinear fractional integro-differential equations with $m$-point multi-term fractional order integral boundary conditions. Our results are based on the Banach contraction principle and Krasnoselskii's fixed point theorem.
\end{abstract}

MSC: 26A33; 34B15

Keywords: existence; Caputo fractional derivative; Riemann-Liouville fractional integral; integral boundary value problem

\section{Introduction}

Differential equations of fractional order arise in several research areas of science and engineering, such as physics, chemistry, aerodynamics, electro-dynamics of complex medium, polymer rheology, electrical networks, control of dynamical systems, etc. Fractional differential equations provide an excellent tool for description of memory and hereditary properties of various materials and processes. Some recent contributions to the theory of fractional differential equations and its applications can be seen in [1-5].

Recently, many researchers have given attention to the existence of solutions of the initial and boundary value problems for fractional differential equations. There are some papers that have studied the existence of solutions to boundary value problems with twopoint, three-point, multi-point or integral boundary conditions. See for examples [6-30]. However, to the best of the authors' knowledge, there are only a few papers that consider nonlinear fractional differential equations with nonlocal fractional order integral boundary conditions, see [31-33].

In this article, we study the existence and uniqueness of solutions for the following nonlinear fractional integro-differential equation with $m$-point multi-term fractional order integral boundary condition:

$$
\begin{aligned}
& { }^{c} D^{q} x(t)=f\left(t, x(t),\left(\psi^{v} x\right)(t)\right), \quad t \in[0, T], q \in(1,2], \\
& x(0)=0, \quad x(T)=\left.\sum_{i=1}^{m-1} \sum_{j=1}^{n_{i}} \alpha_{j, i}\left[I^{p_{j, i}} x\right]\right|_{\xi_{i-1}} ^{\xi_{i}},
\end{aligned}
$$

C 2012 Sudsutad and Tariboon; licensee Springer. This is an Open Access article distributed under the terms of the Creative Commons Attribution License (http://creativecommons.org/licenses/by/2.0), which permits unrestricted use, distribution, and reproduction in any medium, provided the original work is properly cited. 
where ${ }^{c} D^{q}$ denotes the Caputo fractional derivative of order $q$, $\left.\left[I^{p_{, i,}} x\right]\right|_{\xi_{i-1}} ^{\xi_{i}}=\left[I^{p_{j, i}} x\right]\left(\xi_{i}\right)-$ $\left[I^{p_{j, i}} x\right]\left(\xi_{i-1}\right), I^{p_{j, i}}$ is the Riemann-Liouville fractional integral of order $p_{j, i}>0, j=1,2, \ldots, n_{i}$, $n_{i} \in N=\{1,2, \ldots\}, i=1,2, \ldots, m-1,0=\xi_{0}<\xi_{1}<\xi_{2}<\cdots<\xi_{m-2}<\xi_{m-1}=T, f:[0, T] \times X \times$ $X \rightarrow X$, for $\phi:[0, T] \times[0, T] \rightarrow[0, \infty)$,

$$
\left(\psi^{v} x\right)(t)=\left[I^{v} \phi x\right](t)=\frac{1}{\Gamma(v)} \int_{0}^{t}(t-s)^{\nu-1} \phi(t, s) x(s) d s, \quad v>0,
$$

and $\alpha_{j, i} \in R$ is such that $\left.\sum_{i=1}^{m-1} \sum_{j=1}^{n_{i}} \alpha_{j, i} \frac{\xi_{j, i}^{p_{j, i}+1}-\xi_{i-1}^{p_{j i}+1}}{\Gamma\left(p_{j, i}+2\right)}\right) \neq T$. Let $(X,\|\cdot\|)$ be a Banach space and $\mathcal{C}=C([0, T], X)$ denote the Banach space of all continuous functions from $[0, T] \rightarrow X$ endowed with a topology of uniform convergence with the norm denoted by $\|\cdot\|_{\mathcal{C}}$.

We note that if $m=2$, then condition (1.2) reduces to the two-point multi-term fractional integral boundary condition

$$
x(0)=0, \quad x(T)=\sum_{j=1}^{n_{1}} \alpha_{j, 1}\left[I^{p_{j, 1}} x\right](T), \quad n_{1} \in N=\{1,2, \ldots\} .
$$

If $n_{i}=1, i=1,2, \ldots, m-1$, then condition (1.2) reduces to the $m$-point fractional integral boundary condition

$$
x(0)=0, \quad x(T)=\left.\sum_{i=1}^{m-1} \alpha_{1, i}\left[I^{p_{1, i}} x\right]\right|_{\xi_{i-1}} ^{\xi_{i}} .
$$

Moreover, if $p_{1, i}=1$ and $\alpha_{1, i}=\alpha_{i}$ for $i=1,2, \ldots, m-1$, then condition (1.3) reduces to the usual $m$-point integral boundary condition

$$
x(0)=0, \quad x(T)=\alpha_{1} \int_{0}^{\xi_{1}} x(s) d s+\alpha_{2} \int_{\xi_{1}}^{\xi_{2}} x(s) d s+\cdots+\alpha_{m-1} \int_{\xi_{m-2}}^{T} x(s) d s .
$$

In this case, the boundary condition corresponds to $m-1$ intervals of area under the curve of solution $x(t)$ from $t=\xi_{i-1}$ to $t=\xi_{i}$ for $i=1,2, \ldots, m-1$.

\section{Preliminaries}

Let us recall some basic definitions [2, 4].

Definition 2.1 For a continuous function $f:[0, \infty) \rightarrow \mathbb{R}$, the Caputo derivative of fractional order $q$ is defined as

$$
{ }^{c} D^{q} f(t)=\frac{1}{\Gamma(n-q)} \int_{0}^{t}(t-s)^{n-q-1} f^{(n)}(s) d s, \quad n-1<q<n, n=[q]+1,
$$

provided that $f^{(n)}(t)$ exists, where $[q]$ denotes the integer part of the real number $q$.

Definition 2.2 The Riemann-Liouville fractional integral of order $q$ for a continuous function $f(t)$ is defined as

$$
I^{q} f(t)=\frac{1}{\Gamma(q)} \int_{0}^{t}(t-s)^{q-1} f(s) d s, \quad q>0,
$$

provided that such integral exists. 
Definition 2.3 The Riemann-Liouville fractional derivative of order $q$ for a continuous function $f(t)$ is defined by

$$
D^{q} f(t)=\frac{1}{\Gamma(n-q)}\left(\frac{d}{d t}\right)^{n} \int_{0}^{t}(t-s)^{n-q-1} f(s) d s, \quad n=[q]+1,
$$

provided the right-hand side is pointwise defined on $(0, \infty)$.

Lemma 2.1 (see [2]) Let $q>0$, the general solution of the fractional differential equation

$$
{ }^{c} D^{q} x(t)=0
$$

has the solution

$$
x(t)=k_{0}+k_{1} t+k_{2} t^{2}+\cdots+k_{n-1} t^{n-1},
$$

where $k_{i} \in \mathbb{R}, i=0,1,2, \ldots, n-1(n=[q]+1)$.

In view of Lemma 2.1, it follows that

$$
I^{q c} D^{q} x(t)=x(t)+k_{0}+k_{1} t+k_{2} t^{2}+\cdots+k_{n-1} t^{n-1},
$$

for some $k_{i} \in \mathbb{R}, i=0,1,2, \ldots, n-1(n=[q]+1)$.

We state a result due to Krasnoselskii [34] which is needed to prove the existence of at least one solution of the problem (1.1)-(1.2).

Theorem 2.2 Let $M$ be a closed convex and nonempty subset of a Banach space X. Let A, $B$ be operators such that

(i) $A x+B y \in M$ whenever $x, y \in M$,

(ii) A is compact and continuous,

(iii) $B$ is a contraction mapping.

Then there exists $z \in M$ such that $z=A z+B z$.

Lemma 2.3 Let $\left.\sum_{i=1}^{m-1} \sum_{j=1}^{n_{i}} \alpha_{j, i} \frac{\xi_{i, i}^{p_{j, i}+1}-\xi_{i-1}^{p_{j, i}+1}}{\Gamma\left(p_{j, i}+2\right)}\right) \neq T, 1<q \leq 2$. Then for $h \in C[0, T]$, the problem

$$
\begin{aligned}
& { }^{c} D^{q} x(t)=h(t), \quad 0<t<T, \\
& x(0)=0, \quad x(T)=\left.\sum_{i=1}^{m-1} \sum_{j=1}^{n_{i}} \alpha_{j, i}\left[I^{p_{j, i}} x\right]\right|_{\xi_{i-1}} ^{\xi_{i}},
\end{aligned}
$$

has a unique solution

$$
\begin{aligned}
x(t)= & \frac{1}{\Gamma(q)} \int_{0}^{t}(t-s)^{q-1} h(s) d s \\
& -\frac{t}{\Gamma(q)\left(T-\sum_{i=1}^{m-1} \sum_{j=1}^{n_{i}} \alpha_{j, i} \frac{\xi_{j, i}^{p_{j i}+1}-\xi_{i-1}^{p_{j i}+1}}{\Gamma\left(p_{j, i}+2\right)}\right)} \int_{0}^{T}(T-s)^{q-1} h(s) d s
\end{aligned}
$$




$$
\begin{aligned}
& +\frac{t}{\Gamma(q)\left(T-\sum_{i=1}^{m-1} \sum_{j=1}^{n_{i}} \alpha_{j, i} \frac{\xi_{i}^{p_{j, i}+1}-\xi_{i-1}^{p_{j, i}+1}}{\Gamma\left(p_{j, i}+2\right)}\right)} \sum_{i=1}^{m-1} \sum_{j=1}^{n_{i}} \frac{\alpha_{j, i}}{\Gamma\left(p_{j, i}\right)} \\
& \times\left[\int_{0}^{\xi_{i}} \int_{0}^{s}\left(\xi_{i}-s\right)^{p_{j, i}-1}(s-r)^{q-1} h(r) d r d s\right. \\
& \left.-\int_{0}^{\xi_{i-1}} \int_{0}^{s}\left(\xi_{i-1}-s\right)^{p_{j, i}-1}(s-r)^{q-1} h(r) d r d s\right] .
\end{aligned}
$$

Proof We may apply (2.1) to reduce (2.2) to an equivalent integral equation

$$
x(t)=\frac{1}{\Gamma(q)} \int_{0}^{t}(t-s)^{q-1} h(s) d s-c_{0}-c_{1} t
$$

for some constants $c_{0}, c_{1} \in \mathbb{R}$.

From $x(0)=0$, it follows that $c_{0}=0$. Taking the Riemann-Liouville fractional integral of order $p_{j, i}$ for (2.5), we get

$$
\begin{aligned}
{\left[I^{p_{j, i}} x\right](t) } & =\frac{1}{\Gamma\left(p_{j, i}\right)} \int_{0}^{t}(t-s)^{p_{j, i}-1}\left[\frac{1}{\Gamma(q)} \int_{0}^{s}(s-r)^{q-1} h(r) d r-c_{1} s\right] d s \\
& =\frac{1}{\Gamma\left(p_{j, i}\right) \Gamma(q)} \int_{0}^{t} \int_{0}^{s}(t-s)^{p_{j, i}-1}(s-r)^{q-1} h(r) d r d s-c_{1} \frac{t^{p_{j, i}+1}}{\Gamma\left(p_{j, i}+2\right)}
\end{aligned}
$$

On the other hand, for $0 \leq \xi_{i-1} \leq \xi_{i} \leq T$, we obtain

$$
\begin{aligned}
{\left.\left[I^{p_{j, i}} x\right]\right|_{\xi_{i-1}} ^{\xi_{i}}=} & {\left[I^{p_{j, i}} x\right]\left(\xi_{i}\right)-\left[I^{p_{j, i}} x\right]\left(\xi_{i-1}\right) } \\
= & \frac{1}{\Gamma\left(p_{j, i}\right) \Gamma(q)}\left[\int_{0}^{\xi_{i}} \int_{0}^{s}\left(\xi_{i}-s\right)^{p_{j, i}-1}(s-r)^{q-1} h(r) d r d s\right. \\
& \left.-\int_{0}^{\xi_{i-1}} \int_{0}^{s}\left(\xi_{i-1}-s\right)^{p_{j, i}-1}(s-r)^{q-1} h(r) d r d s\right] \\
& -c_{1}\left(\frac{\xi_{i}^{p_{j, i}+1}-\xi_{i-1}^{p_{j, i}+1}}{\Gamma\left(p_{j, i}+2\right)}\right) .
\end{aligned}
$$

The second condition of (2.3) implies that

$$
\begin{aligned}
\frac{1}{\Gamma(q)} & \sum_{i=1}^{m-1} \sum_{j=1}^{n_{i}} \frac{\alpha_{j, i}}{\Gamma\left(p_{j, i}\right)}\left[\int_{0}^{\xi_{i}} \int_{0}^{s}\left(\xi_{i}-s\right)^{p_{j, i}-1}(s-r)^{q-1} h(r) d r d s\right. \\
& \left.-\int_{0}^{\xi_{i-1}} \int_{0}^{s}\left(\xi_{i-1}-s\right)^{p_{j, i}-1}(s-r)^{q-1} h(r) d r d s\right] \\
& -c_{1} \sum_{i=1}^{m-1} \sum_{j=1}^{n_{i}} \alpha_{j, i}\left(\frac{\xi_{i}^{p_{j, i}+1}-\xi_{i-1}^{p_{j, i}+1}}{\Gamma\left(p_{j, i}+2\right)}\right) \\
= & \frac{1}{\Gamma(q)} \int_{0}^{T}(T-s)^{q-1} h(s) d s-c_{1} T .
\end{aligned}
$$


Thus,

$$
\begin{aligned}
c_{1}= & \frac{1}{\Gamma(q)\left(T-\sum_{i=1}^{m-1} \sum_{j=1}^{n_{i}} \alpha_{j, i} \frac{\xi_{j i, i}^{p_{j i}}{ }_{-\xi}^{p_{j, i}+1}}{\Gamma\left(p_{j, i}+2\right)}\right)} \int_{0}^{T}(T-s)^{q-1} h(s) d s \\
& -\frac{1}{\Gamma(q)\left(T-\sum_{i=1}^{m-1} \sum_{j=1}^{n_{i}} \alpha_{j, i} \frac{\xi_{j, i}^{p_{j}+1}-\xi_{i-1}^{p_{j, i}+1}}{\Gamma\left(p_{j, i}+2\right)}\right)} \sum_{i=1}^{m-1} \sum_{j=1}^{n_{i}} \frac{\alpha_{j, i}}{\Gamma\left(p_{j, i}\right)} \\
& \times\left[\int_{0}^{\xi_{i}} \int_{0}^{s}\left(\xi_{i}-s\right)^{p_{j, i}-1}(s-r)^{q-1} h(r) d r d s\right. \\
& \left.-\int_{0}^{\xi_{i-1}} \int_{0}^{s}\left(\xi_{i-1}-s\right)^{p_{j, i}-1}(s-r)^{q-1} h(r) d r d s\right] .
\end{aligned}
$$

Substituting the values of $c_{0}$ and $c_{1}$ in (2.5), we obtain the solution (2.4).

In the following, for the sake of convenience, set

$$
\begin{aligned}
& \lambda=T-\sum_{i=1}^{m-1} \sum_{j=1}^{n_{i}} \alpha_{j, i} \frac{\xi_{i, i}^{p_{j, 1}}-\xi_{i-1}^{p_{j, i}+1}}{\Gamma\left(p_{j, i}+2\right)} \neq 0, \\
& \Omega=\frac{T^{q}}{\Gamma(q+1)}+\frac{T^{q+1}}{|\lambda| \Gamma(q+1)}+\frac{T}{|\lambda|} \sum_{i=1}^{m-1} \sum_{j=1}^{n_{i}} \alpha_{j, i} \frac{\xi_{i}^{q+p_{j, i}}-\xi_{i-1}^{q+p_{j, i}}}{\Gamma\left(q+p_{j, i}+1\right)} .
\end{aligned}
$$

We denote $B(\cdot, \cdot)$ as the standard beta function such that

$$
B(b+1, a)=\int_{0}^{1}(1-s)^{a-1} s^{b} d s=\frac{\Gamma(a) \Gamma(b+1)}{\Gamma(a+b+1)} .
$$

\section{Main results}

Now we are in the position to establish the main result. Our first result is based on Banach's fixed point theorem.

Theorem 3.1 Assume that $f:[0, T] \times X \times X \rightarrow X$ is jointly continuous and maps bounded subsets of $[0, T] \times X \times X$ in to relatively compact subsets of $X$, and $\phi:[0, T] \times[0, T] \rightarrow$ $[0, \infty)$ is continuous with $\phi_{0}=\max \{\phi(t, s):(t, s) \in[0, T] \times[0, T]\}$. In addition, suppose that there exist positive constants $L_{1}, L_{2}$ such that

$\left(H_{1}\right)\left\|f\left(t, x(t),\left(\psi^{v} x\right)(t)\right)-f\left(t, y(t),\left(\psi^{v} y\right)(t)\right)\right\| \leq L_{1}\|x-y\|+L_{2}\left\|\left(\psi^{v} x\right)-\left(\psi^{v} y\right)\right\|$, for all $t \in$ $[0, T], x, y \in X$,

$\left(H_{2}\right) \Lambda=:\left(L_{1}+\frac{L_{2} \phi_{0} T^{v}}{\Gamma(v+1)}\right) \Omega<1$, where $\Omega$ is defined by (2.7).

Then the problem (1.1)-(1.2) has a unique solution.

Proof We transform the BVP (1.1)-(1.2) into a fixed point problem. In view of Lemma 2.3 and (2.6), we consider the operator $F: \mathcal{C} \rightarrow \mathcal{C}$ defined by

$$
\begin{aligned}
(F x)(t)= & \frac{1}{\Gamma(q)} \int_{0}^{t}(t-s)^{q-1} f\left(s, x(s),\left(\psi^{v} x\right)(s)\right) d s \\
& -\frac{t}{\lambda \Gamma(q)} \int_{0}^{T}(T-s)^{q-1} f\left(s, x(s),\left(\psi^{v} x\right)(s)\right) d s
\end{aligned}
$$




$$
\begin{aligned}
& +\frac{t}{\lambda \Gamma(q)} \sum_{i=1}^{m-1} \sum_{j=1}^{n_{i}} \frac{\alpha_{j, i}}{\Gamma\left(p_{j, i}\right)} \\
& \times\left[\int_{0}^{\xi_{i}} \int_{0}^{s}\left(\xi_{i}-s\right)^{p_{j, i}-1}(s-r)^{q-1} f\left(r, x(r),\left(\psi^{v} x\right)(r)\right) d r d s\right. \\
& \left.-\int_{0}^{\xi_{i-1}} \int_{0}^{s}\left(\xi_{i-1}-s\right)^{p_{j, i}-1}(s-r)^{q-1} f\left(r, x(r),\left(\psi^{\nu} x\right)(r)\right) d r d s\right],
\end{aligned}
$$

for $t \in[0, T]$. By setting $\sup _{t \in[0, T]}\|f(t, 0,0)\|=M$, and choosing

$$
r \geq \frac{M \Omega}{1-\Lambda},
$$

we will show that $F B_{r} \subset B_{r}$, where $B_{r}=\{x \in \mathcal{C}:\|x\| \leq r\}$. Then, for $x \in B_{r}$, we have

$$
\begin{aligned}
& \|(F x)(t)\| \leq \frac{1}{\Gamma(q)} \int_{0}^{t}(t-s)^{q-1}\left\|f\left(s, x(s),\left(\psi^{v} x\right)(s)\right)\right\| d s \\
& +\frac{T}{|\lambda| \Gamma(q)} \int_{0}^{T}(T-s)^{q-1}\left\|f\left(s, x(s),\left(\psi^{v} x\right)(s)\right)\right\| d s \\
& +\frac{T}{|\lambda| \Gamma(q)} \sum_{i=1}^{m-1} \sum_{j=1}^{n_{i}} \frac{\alpha_{j, i}}{\Gamma\left(p_{j, i}\right)} \\
& \times\left[\int_{0}^{\xi_{i}} \int_{0}^{s}\left(\xi_{i}-s\right)^{p_{j, i}-1}(s-r)^{q-1}\left\|f\left(r, x(r),\left(\psi^{v} x\right)(r)\right)\right\| d r d s\right. \\
& \left.-\int_{0}^{\xi_{i-1}} \int_{0}^{s}\left(\xi_{i-1}-s\right)^{p_{j, i}-1}(s-r)^{q-1}\left\|f\left(r, x(r),\left(\psi^{\nu} x\right)(r)\right)\right\| d r d s\right] \\
& \leq \frac{1}{\Gamma(q)} \int_{0}^{t}(t-s)^{q-1}\left(\left\|f\left(s, x(s),\left(\psi^{v} x\right)(s)\right)-f(s, 0,0)\right\|+\|f(s, 0,0)\|\right) d s \\
& +\frac{T}{|\lambda| \Gamma(q)} \int_{0}^{T}(T-s)^{q-1}\left(\left\|f\left(s, x(s),\left(\psi^{v} x\right)(s)\right)-f(s, 0,0)\right\|+\|f(s, 0,0)\|\right) d s \\
& +\frac{T}{|\lambda| \Gamma(q)} \sum_{i=1}^{m-1} \sum_{j=1}^{n_{i}} \frac{\alpha_{j, i}}{\Gamma\left(p_{j, i}\right)}\left[\int_{0}^{\xi_{i}} \int_{0}^{s}\left(\xi_{i}-s\right)^{p_{j, i}-1}(s-r)^{q-1}\right. \\
& \times\left(\left\|f\left(r, x(r),\left(\psi^{v} x\right)(r)\right)-f(s, 0,0)\right\|+\|f(s, 0,0)\|\right) d r d s \\
& -\int_{0}^{\xi_{i-1}} \int_{0}^{s}\left(\xi_{i-1}-s\right)^{p_{j, i}-1}(s-r)^{q-1} \\
& \left.\times\left(\left\|f\left(r, x(r),\left(\psi^{v} x\right)(r)\right)-f(s, 0,0)\right\|+\|f(s, 0,0)\|\right) d r d s\right] \\
& \leq \frac{1}{\Gamma(q)}\left[\left(L_{1}+\frac{L_{2} \phi_{0} T^{v}}{\Gamma(v+1)}\right) r+M\right] \int_{0}^{t}(t-s)^{q-1} d s \\
& +\frac{T}{|\lambda| \Gamma(q)}\left[\left(L_{1}+\frac{L_{2} \phi_{0} T^{v}}{\Gamma(v+1)}\right) r+M\right] \int_{0}^{T}(T-s)^{q-1} d s \\
& +\frac{T}{|\lambda| \Gamma(q)}\left[\left(L_{1}+\frac{L_{2} \phi_{0} T^{v}}{\Gamma(v+1)}\right) r+M\right] \\
& \times \sum_{i=1}^{m-1} \sum_{j=1}^{n_{i}} \frac{\alpha_{j, i}}{\Gamma\left(p_{j, i}\right)}\left[\int_{0}^{\xi_{i}} \int_{0}^{s}\left(\xi_{i}-s\right)^{p_{j, i}-1}(s-r)^{q-1} d r d s\right.
\end{aligned}
$$




$$
\begin{aligned}
& \left.-\int_{0}^{\xi_{i-1}} \int_{0}^{s}\left(\xi_{i-1}-s\right)^{p_{j, i}-1}(s-r)^{q-1} d r d s\right] \\
\leq & \left(\left(L_{1}+\frac{L_{2} \phi_{0} T^{v}}{\Gamma(v+1)}\right) r+M\right)\left[\frac{T^{q}}{\Gamma(q+1)}+\frac{T^{q+1}}{|\lambda| \Gamma(q+1)}\right. \\
& \left.+\frac{T}{|\lambda| \Gamma(q+1)} \sum_{i=1}^{m-1} \sum_{j=1}^{n_{i}} \frac{\alpha_{j, i}}{\Gamma\left(p_{j, i}\right)}\left(\xi_{i}^{q+p_{j, i}} B\left(q+1, p_{j, i}\right)-\xi_{i-1}^{q+p_{j, i}} B\left(q+1, p_{j, i}\right)\right)\right] \\
= & \left(\left(L_{1}+\frac{L_{2} \phi_{0} T^{v}}{\Gamma(v+1)}\right) r+M\right)\left[\frac{T^{q}}{\Gamma(q+1)}+\frac{T^{q+1}}{|\lambda| \Gamma(q+1)}\right. \\
& \left.+\frac{T}{|\lambda|} \sum_{i=1}^{m-1} \sum_{j=1}^{n_{i}} \alpha_{j, i} \frac{\xi_{i}^{q+p_{j, i}}-\xi_{i-1}^{q+p_{j, i}}}{\Gamma\left(q+p_{j, i}+1\right)}\right]=\Omega\left[\left(L_{1}+\frac{L_{2} \phi_{0} T^{v}}{\Gamma(v+1)}\right) r+M\right] \leq r .
\end{aligned}
$$

In the following, from $\left(H_{1}\right)$ for $x, y \in \mathcal{C}$ and for each $t \in[0, T]$, we have

$$
\begin{aligned}
&\|(F x)(t)-(F y)(t)\| \\
& \leq \frac{1}{\Gamma(q)} \int_{0}^{t}(t-s)^{q-1}\left\|f\left(s, x(s),\left(\psi^{v} x\right)(s)\right)-f\left(s, y(s),\left(\psi^{v} y\right)(s)\right)\right\| d s \\
&+\frac{T}{|\lambda| \Gamma(q)} \int_{0}^{T}(T-s)^{q-1}\left\|f\left(s, x(s),\left(\psi^{v} x\right)(s)\right)-f\left(s, y(s),\left(\psi^{v} y\right)(s)\right)\right\| d s \\
&+\frac{T}{|\lambda| \Gamma(q)} \sum_{i=1}^{m-1} \sum_{j=1}^{n_{i}} \frac{\alpha_{j, i}}{\Gamma\left(p_{j, i}\right)} \\
& \times\left[\int_{0}^{\xi_{i}} \int_{0}^{s}\left(\xi_{i}-s\right)^{p_{j, i}-1}(s-r)^{q-1}\left\|f\left(r, x(r),\left(\psi^{v} x\right)(r)\right)-f\left(r, y(r),\left(\psi^{v} y\right)(r)\right)\right\| d r d s\right. \\
&\left.-\int_{0}^{\xi_{i-1}} \int_{0}^{s}\left(\xi_{i-1}-s\right)^{p_{j, i}-1}(s-r)^{q-1}\left\|f\left(r, x(r),\left(\psi^{v} x\right)(r)\right)-f\left(r, y(r),\left(\psi^{v} y\right)(r)\right)\right\| d r d s\right] \\
& \leq \frac{1}{\Gamma(q)}\left(L_{1}+\frac{L_{2} \phi_{0} T^{v}}{\Gamma(v+1)}\right)\|x-y\|_{\mathcal{C}} \int_{0}^{t}(t-s)^{q-1} d s \\
&+\frac{T}{|\lambda| \Gamma(q)}\left(L_{1}+\frac{L_{2} \phi_{0} T^{v}}{\Gamma(v+1)}\right)\|x-y\|_{\mathcal{C}} \int_{0}^{T}(T-s)^{q-1} d s \\
&+\frac{T}{|\lambda| \Gamma(q)}\left(L_{1}+\frac{L_{2} \phi_{0} T^{v}}{\Gamma(v+1)}\right)\|x-y\|_{\mathcal{C}} \\
& \times \sum_{i=1}^{m-1} \sum_{j=1}^{n_{i}} \frac{\alpha_{j, i}}{\Gamma\left(p_{j, i}\right)}\left[\int_{0}^{\xi_{i}} \int_{0}^{s}\left(\xi_{i}-s\right)^{p_{j, i}-1}(s-r)^{q-1} d r d s\right. \\
&\left.-\int_{0}^{\xi_{i-1}} \int_{0}^{s}\left(\xi_{i-1}-s\right)^{p_{j, i}-1}(s-r)^{q-1} d r d s\right] \\
& \leq\left.\frac{L_{2} \phi_{0} T^{v}}{\Gamma(v+1)}\right) \Omega\|x-y\|_{\mathcal{C}}=\Lambda\|x-y\|_{\mathcal{C}} .
\end{aligned}
$$

By $\left(H_{2}\right), \Lambda<1$, therefore, $F$ is a contraction. Hence, by the Banach fixed point theorem, we get that $F$ has a fixed point which is a unique solution of the problem (1.1)-(1.2).

Our second result is based on Krasnoselskii's fixed point theorem. 
Theorem 3.2 Assume that $\left(H_{1}\right)$ and $\left(H_{2}\right)$ hold with

$$
\left\|f\left(t, x(t),\left(\psi^{v} x\right)(t)\right)\right\| \leq \mu(t)
$$

for all $\left(t, x,\left(\psi^{v} x\right)\right) \in[0, T] \times X \times X$, where $\mu \in L^{1}\left([0, T], R^{+}\right)$.

Then the BVP (1.1)-(1.2) has at least one solution on $[0, T]$.

Proof First, we choose

$$
r \geq\|\mu\|_{L^{1}} \Omega
$$

where $\Omega$ is defined by (2.7), and consider $B_{r}=\{x \in \mathcal{C}:\|x\| \leq r\}$. On $B_{r}$ we define the operators $S$ and $U$ as follows:

$$
\begin{aligned}
(S x)(t)= & \frac{1}{\Gamma(q)} \int_{0}^{t}(t-s)^{q-1} f\left(s, x(s),\left(\psi^{v} x\right)(s)\right) d s \\
(U x)(t)= & \frac{t}{\lambda \Gamma(q)} \sum_{i=1}^{m-1} \sum_{j=1}^{n_{i}} \frac{\alpha_{j, i}}{\Gamma\left(p_{j, i}\right)} \\
& \times\left[\int_{0}^{\xi_{i}} \int_{0}^{s}\left(\xi_{i}-s\right)^{p_{j, i}-1}(s-r)^{q-1} f\left(r, x(r),\left(\psi^{v} x\right)(r)\right) d r d s\right. \\
& \left.-\int_{0}^{\xi_{i-1}} \int_{0}^{s}\left(\xi_{i-1}-s\right)^{p_{j, i}-1}(s-r)^{q-1} f\left(r, x(r),\left(\psi^{v} x\right)(r)\right) d r d s\right] \\
& -\frac{t}{\lambda \Gamma(q)} \int_{0}^{T}(T-s)^{q-1} f\left(s, x(s),\left(\psi^{v} x\right)(s)\right) d s .
\end{aligned}
$$

Now we shall show that $S+U$ has a fixed point in $B_{r}$. For $x, y \in B_{r}$, we have

$$
\begin{aligned}
\|S x+U y\| \leq & \|\mu\|_{L^{1}}\left(\frac{1}{\Gamma(q)} \int_{0}^{t}(t-s)^{q-1} d s+\frac{T}{|\lambda| \Gamma(q)} \int_{0}^{T}(T-s)^{q-1} d s\right. \\
& +\frac{T}{|\lambda| \Gamma(q)} \sum_{i=1}^{m-1} \sum_{j=1}^{n_{i}} \frac{\alpha_{j, i}}{\Gamma\left(p_{j, i}\right)} \\
& \times\left[\int_{0}^{\xi_{i}} \int_{0}^{s}\left(\xi_{i}-s\right)^{p_{j, i}-1}(s-r)^{q-1} d r d s\right. \\
& \left.\left.-\int_{0}^{\xi_{i-1}} \int_{0}^{s}\left(\xi_{i-1}-s\right)^{p_{j, i}-1}(s-r)^{q-1} d r d s\right]\right) \\
\leq & \|\mu\|_{L^{1}} \Omega \leq r .
\end{aligned}
$$

Therefore, $S x+U y \in B_{r}$.

From assumptions $\left(H_{1}\right)$ and $\left(H_{2}\right)$ for $x, y \in \mathcal{C}$ and for each $t \in[0, T]$, we get that

$$
\|(U x)(t)-(U y)(t)\| \leq \Lambda\|x-y\|_{\mathcal{C}} .
$$

Hence, $U$ is a contraction mapping.

Next, we show that $S$ is compact and continuous. 
The continuity of $f$ implies that the operator $S$ is continuous. By using condition (3.2), we have that $S$ is uniformly bounded on $B_{r}$ as

$$
\|S x\| \leq \frac{\|\mu\|_{L^{1}} T^{q}}{\Gamma(q+1)}
$$

Furthermore, in view of $\left(H_{1}\right)$, we define $\sup _{\left(t, x,\left(\psi^{v} x\right)\right) \in[0, T] \times B_{r} \times B_{r}}\left\|f\left(t, x,\left(\psi^{v} x\right)\right)\right\|=\bar{f}$, and consequently we get

$$
\begin{aligned}
\left\|(S x)\left(t_{2}\right)-(S x)\left(t_{1}\right)\right\| \\
=\| \frac{1}{\Gamma(q)} \int_{0}^{t_{2}}\left(t_{2}-s\right)^{q-1} f\left(s, x(s),\left(\psi^{v} x\right)(s)\right) d s \\
\quad-\frac{1}{\Gamma(q)} \int_{0}^{t_{1}}\left(t_{1}-s\right)^{q-1} f\left(s, x(s),\left(\psi^{v} x\right)(s)\right) d s \| \\
\leq \frac{1}{\Gamma(q)} \int_{0}^{t_{1}}\left(\left(t_{2}-s\right)^{q-1}-\left(t_{1}-s\right)^{q-1}\right)\left\|f\left(s, x(s),\left(\psi^{v} x\right)(s)\right)\right\| d s \\
\quad+\frac{1}{\Gamma(q)} \int_{t_{1}}^{t_{2}}\left(t_{2}-s\right)^{q-1}\left\|f\left(s, x(s),\left(\psi^{v} x\right)(s)\right)\right\| d s \\
\leq \frac{\bar{f}}{\Gamma(q+1)}\left|2\left(t_{2}-t_{1}\right)^{q}+t_{2}^{q}-t_{1}^{q}\right|,
\end{aligned}
$$

which is independent of $x$. As $t_{2} \rightarrow t_{1}$, the right-hand side of the above inequality tends to zero. So, $S$ is relatively compact on $B_{r}$. Hence, by Arzelá-Ascoli theorem, $S$ is compact on $B_{r}$. Thus, all the assumptions of Theorem 2.2 are satisfied. As a consequence of Theorem 2.2, we have that the boundary value problem (1.1)-(1.2) has at least one solution on $[0, T]$. This completes the proof.

\section{An example}

Example 4.1 Consider the following four-point multi-term fractional integral boundary value problem:

$$
\begin{aligned}
& { }^{c} D^{\frac{3}{2}} x(t)=\frac{1}{(t+9)^{2}} \cdot \frac{|x|}{|x|+1}+\int_{0}^{t} \frac{(t-s)^{-\frac{1}{2}}}{\Gamma\left(\frac{1}{2}\right)} \cdot \frac{e^{-(s-t)}}{81} x(s) d s, \quad t \in[0,3], \\
& x(0)=0, \\
& x(3)=\left.\frac{3}{8}\left[I^{\frac{1}{2}} x\right]\right|_{0} ^{1}-\left.\frac{2}{5}\left[I^{\frac{3}{2}} x\right]\right|_{0} ^{1}+\left.\frac{7}{2}\left[I^{\frac{5}{2}} x\right]\right|_{1} ^{2}-\left.5\left[I^{\frac{1}{2}} x\right]\right|_{2} ^{3}+\left.2\left[I^{\frac{3}{2}} x\right]\right|_{2} ^{3}-\left.3\left[I^{\frac{5}{2}} x\right]\right|_{2} ^{3} .
\end{aligned}
$$

Set $q=3 / 2, m=4, \xi_{0}=0, \xi_{1}=1, \xi_{2}=2, \xi_{3}=T=3, n_{1}=2, p_{1,1}=1 / 2, p_{2,1}=3 / 2, \alpha_{1,1}=3 / 8$, $\alpha_{2,1}=-2 / 5, n_{2}=1, p_{1,2}=5 / 2, \alpha_{1,2}=7 / 2, n_{3}=3, p_{1,3}=1 / 2, p_{2,3}=3 / 2, p_{3,3}=5 / 2, \alpha_{1,3}=-5$, $\alpha_{2,3}=2, \alpha_{3,3}=-3, v=1 / 2, \phi(t, s)=e^{-(s-t)} / 81, f(t, x)=\left(1 /(t+9)^{2}\right)(|x| /(|x|+1))+\left(\psi^{\frac{1}{2}} x\right)(t)$. Since $\left\|f\left(t, x,\left(\psi^{\frac{1}{2}} x\right)\right)-f\left(t, y,\left(\psi^{\frac{1}{2}} y\right)\right)\right\| \leq(1 / 81)\|x-y\|+\left\|\left(\psi^{\frac{1}{2}} x\right)-\left(\psi^{\frac{1}{2}} y\right)\right\|$, then $\left(H_{1}\right)$ and $\left(H_{2}\right)$ are satisfied with

$$
\lambda=T-\sum_{i=1}^{m-1} \sum_{j=1}^{n_{i}} \alpha_{j, i} \frac{\xi_{i}^{p_{j, i}+1}-\xi_{i-1}^{p_{j, i}+1}}{\Gamma\left(p_{j, i}+2\right)} \approx 11.80716 \neq 0,
$$




$$
\begin{gathered}
\Omega=\frac{T^{q}}{\Gamma(q+1)}+\frac{T^{q+1}}{|\lambda| \Gamma(q+1)}+\frac{T}{|\lambda|} \sum_{i=1}^{m-1} \sum_{j=1}^{n_{i}} \alpha_{j, i} \frac{\xi_{i}^{q+p_{j, i}}-\xi_{i-1}^{q+p_{j, i}}}{\Gamma\left(q+p_{j, i}+1\right)} \approx 1.85722, \\
L_{1}=1 / 81, L_{2}=1, \phi_{0}=e^{3} / 81, T^{v}=\sqrt{3}, \Gamma(v+1)=\sqrt{\pi} / 2 . \text { We can show that } \\
\Lambda=\left(L_{1}+\frac{L_{2} \phi_{0} T^{v}}{\Gamma(v+1)}\right) \Omega \approx 0.92300<1 .
\end{gathered}
$$

Hence, by Theorem 3.1, the boundary value problem (4.1)-(4.2) has a unique solution on $[0,3]$.

\section{Competing interests}

The authors declare that they have no competing interests.

\section{Authors' contributions}

All authors contributed equally in this paper. They read and approved the final manuscript.

\section{Acknowledgements}

This research is supported by the Centre of Excellence in Mathematics, the Commission on Higher Education, Thailand.

Received: 19 April 2012 Accepted: 9 August 2012 Published: 30 August 2012

\section{References}

1. Hilfer, R: Fractional Calculus in Physics. World Scientific, Singapore (2000)

2. Kilbas, AA, Srivastava, HM, Trujillo, JJ: Theory and Applications of Fractional Differential Equations. Elsevier, Amsterdam (2006)

3. Mainardi, F: Fractional Calculus and Waves in Linear Viscoelasticity: An Introduction to Mathematical Models. Imperial College Press, Singapore (2010)

4. Podlubny, I: Fractional Differential Equations. Academic Press, New York (1999)

5. Sabatier, J, Agrawal, OP, Machado, JAT (eds.): Advances in Fractional Calculus: Theoretical Developments and Applications in Physics and Engineering. Springer, Dordrecht (2007)

6. Agarwal, RP, Ahmad, B: Existence theory for anti-periodic boundary value problems of fractional differential equations and inclusions. Comput. Math. Appl. 62, 1200-1214 (2011)

7. Ahmad, B, Nieto, JJ: Existence results for nonlinear boundary value problems of fractional integrodifferential equations with integral boundary conditions. Bound. Value Probl. 2009, Article ID 708576 (2009)

8. Ahmad, B, Nieto, JJ: Solvability of nonlinear Langevin equation involving two fractional orders with Dirichlet boundary conditions. Int. J. Differ. Equ. 2010, Article ID 649486 (2010)

9. Ahmad, B, Nieto, JJ, Alsaedi, A, El-Shahed, M: A study of nonlinear Langevin equation involving two fractional orders in different intervals. Nonlinear Anal., Real World Appl. 13, 599-606 (2012)

10. Ahmad, B, Ntouyas, SK, Alsaedi, A: New existence results for nonlinear fractional differential equations with three-point integral boundary conditions. Adv. Differ. Equ. 2011, Article ID 107384 (2011)

11. Ahmad, B, Sivasundaram, S: On four-point nonlocal boundary value problems of nonlinear integro-differential equations of fractional order. Appl. Math. Comput. 217, 480-487 (2010)

12. Ahmad, B, Sivasundaram, S: Theory of fractional differential equations with three-point boundary conditions. Commun. Appl. Anal. 12, 479-484 (2008)

13. Bai, Z, Qiu, T: Existence of positive solution for singular fractional differential equation. Appl. Math. Comput. 215 2761-2767 (2009)

14. Benchohra, M, Hamani, S, Ntouyas, SK: Boundary value problems for differential equations with fractional order. Surv. Math. Appl. 3, 1-12 (2008)

15. Benchohra, M, Hamani, S, Ntouyas, SK: Boundary value problems for differential equations with fractional order and nonlocal conditions. Nonlinear Anal. 71, 2391-2396 (2009)

16. Cabada, A, Wang, G: Positive solutions of nonlinear fractional differential equations with integral boundary value conditions. J. Math. Anal. Appl. 389, 403-411 (2012)

17. Chai, G: Existence results for boundary value problems of nonlinear fractional differential equations. Comput. Math. Appl. 62, 2374-2380 (2011)

18. Cui, Y: Existence results for singular boundary value problem of nonlinear fractional differential equation. Abstr. Appl. Anal. 2011, Article ID 605614 (2011)

19. Hamani, S, Benchohra, M, Graef, JR: Existence results for boundary-value problems with nonlinear fractional differential inclusions and integral conditions. Electron. J. Differ. Equ. 2010(20), 1-16 (2010)

20. Jia, M, Liu, X: Three nonnegative solutions for fractional differential equations with integral boundary conditions. Comput. Math. Appl. 62, 1405-1412 (2011)

21. Liu, X, Jia, M: Multiple solutions for fractional differential equations with nonlinear boundary conditions. Comput. Math. Appl. 59, 2880-2886 (2010)

22. Nyamoradi, N: Existence of solutions for multi point boundary value problems for fractional differential equations. Arab J. Math. Sci. 18, 165-175 (2012) 
23. Rehman, MU, Khan, RA: Existence and uniqueness of solutions for multi-point boundary value problems for fractional differential equations. Appl. Math. Lett. 23, 1038-1044 (2010)

24. Rehman, MU, Khan, RA, Asif, NA: Three point boundary value problems for nonlinear fractional differential equations. Acta Math. Sci. 31, 1337-1346 (2011)

25. Su, X, Zhang, S: Solutions to boundary-value problems for nonlinear differential equations of fractional order. Electron. J. Differ. Equ. 2009(26), 1-15 (2009)

26. Tian, Y, Zhou, Y: Positive solutions for multipoint boundary value problem of fractional differential equations. J. Appl. Math. Comput. 38, 417-427 (2012)

27. Wang, Y, Liu, L, Wu, Y: Positive solutions for a nonlocal fractional differential equation. Nonlinear Anal. 74, 3599-3605 (2011)

28. Zhao, Y, Sun, S, Han, Z, Li, Q: The existence of multiple positive solutions for boundary value problems of nonlinear fractional differential equations. Commun. Nonlinear Sci. Numer. Simul. 16, 2086-2097 (2011)

29. Zhong, W, Lin, W: Nonlocal and multi-point boundary value problem for fractional differential equations. Comput. Math. Appl. 59, 1345-1351 (2010)

30. Zhou, WX, Chu, YD: Existence of solutions for fractional differential equations with multi-point boundary conditions. Commun. Nonlinear Sci. Numer. Simul. 17, 1142-1148 (2012)

31. Ahmad, B, Nieto, JJ: Riemann-Liouville fractional integro-differential equations with fractional nonlocal integral boundary conditions. Bound. Value Probl. 2011, 36 (2011)

32. Sudsutad, W, Tariboon, J: Boundary value problems for fractional differential equations with three-point fractional integral boundary conditions. Adv. Differ. Equ. 2012, 93 (2012)

33. Wang, G, Liu, W, Ren, C: Existence of solutions for multi-point nonlinear differential equations of fractional orders with integral boundary conditions. Electron. J. Differ. Equ. 2012(54), 1-10 (2012)

34. Krasnoselskii, MA: Two remarks on the method of successive approximations. Usp. Mat. Nauk 10, 123-127 (1955)

doi:10.1186/1687-2770-2012-94

Cite this article as: Sudsutad and Tariboon: Existence results of fractional integro-differential equations with $m$-point multi-term fractional order integral boundary conditions. Boundary Value Problems 2012 2012:94.

\section{Submit your manuscript to a SpringerOpen ${ }^{\ominus}$ journal and benefit from:}

- Convenient online submission

Rigorous peer review

- Immediate publication on acceptance

Open access: articles freely available online

- High visibility within the field

- Retaining the copyright to your article 